\title{
Solution for Optimal Design Parameters of Desktop Based on \\ Multi-objective Programming
}

\author{
Han Dong \\ School of Computer Science. North China Electric Power University, Baoding, China. \\ 411135604@qq.com
}

\begin{abstract}
This paper is selected from Question B of the 2014 Chinese college student mathematical modeling contest. A multi-objective programming model was established to find the optimal processing parameters. In order to externalize the compression capability of the desktop, we use the Euler equation of critical force to find the relationship between the material and the size of the force. We set three goals to our creative folding table: stability, simple producing process and least needed material. Besides, the stability of products was put the priority in the solving process of producing parameters.
\end{abstract}

Keywords: Multi-objective programming; Euler equation of Critical force.

\section{The Establishment of the Stability of Optimal Model}

The question requests Stability, simple producing process and least needed material are the three major goals to be used to determine the optimal parameters under the condition of a given table height and size of the desktop. So the first step should be establish the mathematical model of sub-target and solve multi-objective programming under the restriction of multiple targets.

The good stability of the table is reflected in maintaining stable state in the case of desktop under high gravity. Only the four sticks at the bottom of the table contact with the ground after desktop launched. The physical structure of the table is axisymmetric. That is to say force evenly dispersed to the four sticks on the edge of the table. According to the physical state, we can establish the force analysis model and the edge of the table legs' force analysis model under the optimal stability target for further discussion.

\subsection{The Overall Stress Analysis Model of the Table.}

Under the control of hinge, the bending degree of four legs may determine the stability of the table to a certain extent when the folding table fully expanded and placed in a horizontal plane in a stable static state. The extent of tilt is determined by the Angle of legs and ground. Supposing the desktop as a stress analysis plane, Viewed from the $\mathrm{Y}$ axis direction in the spatial reference frame $S$, we can abstract the stress analysis of the table as figure 1.

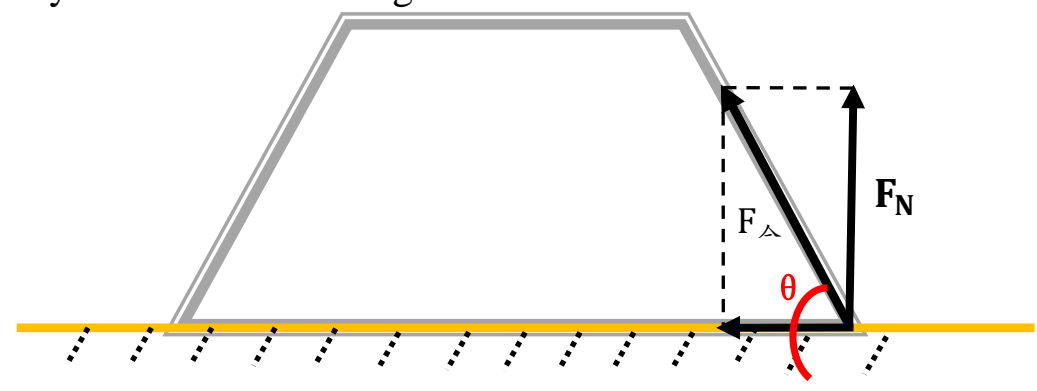

\author{
s
}

Figure 1 stress analysis of the table

In figure 1: $F_{N}$ is the support of the ground. According to physical space and structure of the 
table, The total weight of the table is four times as large as $F_{N} ; f$ is the static friction force between the table leg with the ground; $F_{\text {sum }}$ is the resultant force. According to stress analysis, we can get a series of mechanical relations constraint conditions:

$$
\left\{\begin{array}{l}
F_{N}=\frac{1}{4} G \\
f_{\max }=\mu F_{N}, f<f_{\max } \\
\tan \theta=F_{N} / f
\end{array}\right.
$$

In the formula: $f_{\max }$ is the maximum static friction force; $\theta$ is the Angle between the stick and horizontal plane; $\mu$ is the friction coefficient of the ground.

According to the above constraint condition, we get a constraint condition of the stability of the table: $\tan \theta>\frac{1}{\mu}$

The static friction coefficient of horizontal ground must meet at least 0.5 safety requirement according to the regulation of international standards of slippery ground. So $\mu$ is 0.5 . Get the range of $\theta$ in the last: $63.43^{\circ}<\theta<116.56^{\circ}$

\subsection{The Establishment of the Force Analysis Model of Table Leg.}

Considering the total capacity (including the gravity of the table and the pressure of things on the table) of the whole table is on the edge of the four sticks, in order to explore the folding table maximum bearing capacity under stationary state, we must to solve the biggest bearing critical force at the edge of the thin stick. Due to the stress of the four sticks almost unanimously in bearing, we draw a thin stick to analysis.

Under the table body stationary states, we set the table leg abstract into a long thin rod as figure 2: (Due to the need to explore the critical force of these four sticks, they should not be regarded as rigid body in the critical state)

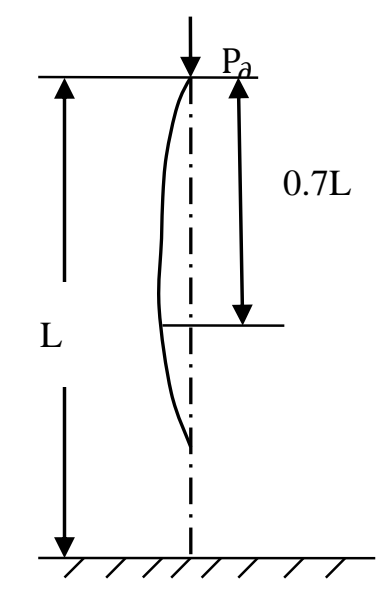

Figure 2 critical force analysis diagram

Then according to the physical condition of the thin stick: At one end hinged (with desktop tablet with a hinge), one end is fixed (solid grounding). On the basis of Euler formula of constraints to critical force the biggest bear ability of the thin batten and according to physical stress constraint 
conditions of the table leg, the stress constraint model [2] is established:

$$
\left\{\begin{array}{l}
P_{\alpha}=\frac{\pi^{2} E I}{(0.7 L)^{2}} \\
F_{\text {sum_max }}=\frac{1}{4} \times G \times \sqrt{1+\mu^{2}} \\
F_{\text {sum_max }} \leq P_{\partial} \\
l \sin \theta=h
\end{array}\right.
$$

In the formula: $P_{\alpha}$ is the critical loading capacity of the thin batten; $F_{\text {sum_max }}$ is the biggest support by the ground the thin batten; $E$ is the material's elastic modulus; $I$ is moment inertia of rod's cross section; $l$ is the length of the thin batten.

\section{The Optimal Model of Simple Processing}

The question require parts processing as easy as possible, that is to say the total length of slots as short as possible, but at the same time to meet the dynamic range of folding rebar.

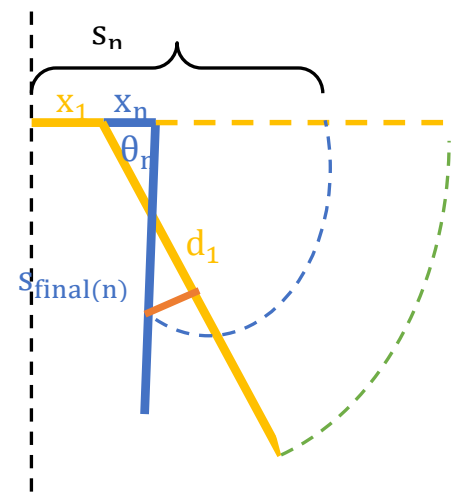

Figure 3 Relative positions of the batten analysis diagram

The dotted line in figure 3 is the sliding route of rebar. According to the figure, rebar mobile location should be beyond the range of the fixed hinge in the physical motion state. On this basis, using cosine theorem, we establish the following mathematical constraint equation:

$$
s_{\text {final }(n)}=\sqrt{d_{1}^{2}+\left(x_{n}-x_{1}\right)^{2}-2 d_{1}\left(x_{n}-x_{1}\right) \cos \theta}
$$

The objective function

$$
\min \sum_{1}^{n}\left[\left(s_{\text {final }(n)}+x_{n}\right)-\left(s_{1}+x_{1}\right)\right]
$$

Constraint condition

$$
\begin{aligned}
& s_{\text {final }(n)}<\frac{b}{2}-x_{n} \\
& s_{n}<x_{n}
\end{aligned}
$$

In the formula: $s_{\text {final }(n)}$ is the distance from the hinge to the rebar after folding in the stick n. $x_{n}$ is the distance from the center axis to the hinge of the wood $n . b$ is the length of the rectangular plate.

Due to the diameter of the circular plate is determined and the width of the rectangular plate is also 
sure to determine, meeting the minimum material means letting the rectangular plate as short as possible under the given limiting conditions.

\section{The Establishment of Multi-objective Programming Model}

\subsection{The Overall Plan for the Multi-objective Function.}

To solve the optimal design and processing parameter of the folding table, with processing products more stable, more convenient (minimum total slot length), less wood used for three objective function first of all, we establish a multi-objective programming model as follows:

Goal of strongest stability

$$
\max G=4 \times \frac{\pi^{2} E I}{0.7 \mu} \sin \theta_{1}, I=\frac{1}{12} d \times h_{0}^{2}
$$

Goal of simple processing

$$
\min d_{\text {sum }}=\sum_{i=1}^{n} d_{i}
$$

Goal of minimize material

$$
\min l_{\text {sum }}=2 \times\left(l_{1}+x_{1}\right)
$$

Restricted condition

$$
\begin{aligned}
& 63.43^{\circ}<\theta<116.56^{\circ}, \quad l_{1} \sin \theta=h, x_{i}+s_{\text {final }(i)}<x_{1}+l_{1}, \\
& y_{i-1}<x_{i}<y_{i}, 0<x_{1}<y_{1}, \quad i=1,2, \ldots, n
\end{aligned}
$$

$d_{i}$ is the length of slot of the i-th stick; $y_{i}$ is the ordinate of the intersection of line and circular;

$d_{\text {sum }}$ is the total length of the slot of all the stick; $\theta$ is the angle of the first stick and horizontal.

We used the given size (the height is $70 \mathrm{~cm}$ of the table, the diameter of the desktop was $80 \mathrm{~cm}$ ) to limit the design goal and solve the three design processing parameters including the size of the board, the location of the rebar and the length of the slot on the basic of the establishment of overall multiobjective programming model. The thickness of the board and the width of the wood still continue to use the standard in the first question $3 \mathrm{~cm}$ and $2.5 \mathrm{~cm}$ respectively. According to the above, a group of wood: $n=80 / 2.5=32$.

Due to the product positioning for creative folding tables are diverse, we can not immediately determine the priorities and the weight of each target to solve the optimal parameter. The stability of products was put the priority in the solving process of producing parameters in this paper.

\section{Conclusion}

In order to make the table more stable, that is, under the condition of fixed design and processing parameters, table 1 can withstand the pressure as much as possible. We established a multi-objective priority under the priority: primary goal: stability; the second goal: maximize material savings; the third goal: maximum simplify the producing process. By using LINGO to solve the problem, the design and processing parameters are as table 1. 
Table 1 Statistics of the length of the slot and the length of the stick under the restriction of stability

\begin{tabular}{ccc|ccc}
\hline $\begin{array}{c}\text { Serial number } \\
\text { of the stick }\end{array}$ & $\begin{array}{c}\text { Length of the } \\
\text { stick }\end{array}$ & $\begin{array}{c}\text { Length of the } \\
\text { slot }\end{array}$ & $\begin{array}{c}\text { Serial number } \\
\text { of the stick }\end{array}$ & $\begin{array}{c}\text { Length of the } \\
\text { stick }\end{array}$ & $\begin{array}{c}\text { Length of the } \\
\text { slot }\end{array}$ \\
\hline 1 & 70.00 & 0.00 & 9 & 47.26 & 30.14 \\
2 & 67.98 & 2.08 & 10 & 45.93 & 32.26 \\
3 & 62.54 & 8.34 & 11 & 44.82 & 34.06 \\
4 & 58.58 & 13.44 & 12 & 43.90 & 35.56 \\
5 & 55.44 & 17.78 & 13 & 43.17 & 36.76 \\
6 & 52.85 & 21.54 & 14 & 42.61 & 37.69 \\
7 & 50.68 & 24.81 & 15 & 42.21 & 38.35 \\
8 & 48.83 & 27.66 & 16 & 41.98 & 38.74 \\
\hline
\end{tabular}

1. Length of the slot and the length of the stick

2. Size of the board $(\mathrm{cm})-163.8 \times 80 \times 3$

3. Location of the rebar $(\mathrm{cm})-43.15952$

4. Critical pressure (N)-923.9524

\section{References}

[1] Guoben Cheng. Discussing of the urgency of developing standards to prevent slipping in China. [J]. Stone. 2008(1).

[2] Changcheng Yin. Proposed algorithm of calculating slender compressive bar' critical pressure [J]. Journal of Hubei Automotive Industries Institute. 2004(02):6-12

[3] Shoukui Si, Mathematical modeling algorithm and its application [M]. National Defense Industry Press, Beijing, 2014.2 